\title{
PHYSICAL MESON-BARYON SCATTERING IN THE CANONICAL ALGEBRA ${ }^{\dagger}$
}

\author{
Shafik J. HAKIM ${ }^{\dagger \dagger}$ \\ Randall Laboratory of Physics, \\ University of Michigan, Ann Arbor, Mich., 48104, USA \\ Received 11 January 1972 \\ (Revised 10 July 1972)
}

\begin{abstract}
A recalculation of the meson-baryon scattering lengths yielded a good agreement with experimental data and a qualitative agreement with a previous calculation of Von Hippel and Kim. The connected matrix element of the singlet member of the SU (3) $\otimes S U(3)$ scalar nonet $\left(3,3^{*}\right)+\left(3^{*}, 3\right)$ between baryon states $\mu_{0}$ was found to be $290 \pm 32 \mathrm{MeV}$ and the pion-nucleon $\sigma$-term $30 \pm 2 \mathrm{MeV}$.
\end{abstract}

Assuming PCAC and canonical field algebra, it was shown by refs. [1-3] that the real part of the symmetric $(+)$ and antisymmetric $(-)$ scattering lengths $a$ for meson-baryon scattering can be written as a sum of the soft-meson terms [4] and a dispersion integral over the imaginary part of the elastic scattering amplitude $T$. This could be used to evaluate $\operatorname{Re} a^{( \pm)}$if the dispersion integral ${ }^{\dagger+f}$ and the $\sigma$ term $\ddagger$ (appearing in the symmetric amplitude) could be evaluated. In the $\sigma$-model [5], it was shown [2] that the $\sigma$-term can be expressed in terms of known SU(3) parameters except one term $\mu_{0}$, which is the connected part of the matrix element of the singlet member of the $\mathrm{SU}(3) \otimes \mathrm{SU}(3)$ scalar nonet $\left(3,3^{*}\right)+\left(3^{*}, 3\right)$ between baryon states. In this note we present the results of a recalculation of the work of ref. [2], namely the real part of the meson-baryon scattering lengths and the pionnucleon $\sigma$-term. However, there are some differences. For, while ref. [2] used a

$\dagger$ Supported in part by the US Atomic Energy Commission.

$\dagger \dagger$ Fellow, Council for Scientific Research, Lebanon.

On leave of absence from Physics Department, Kuwait University, Kuwait. Present address International Centre for Theoretical Physics, PO Box 586, I-34100 Trieste, Italy.

$\dagger \dagger \dagger$ For details see refs. $[1-3]$.

$\ddagger$ The meson-baryon $\sigma$-term $\sigma_{\alpha \beta}$ is defined by

$$
\sigma_{\alpha \beta}=-i \int \mathrm{d}^{4} x \mathrm{e}^{i q \cdot x}\left\langle B\left|\left[\dot{A}_{\beta}^{0}\left(\frac{1}{2} x\right), A_{\alpha}^{0}\left(-\frac{1}{2} x\right)\right]\right| B\right\rangle-\text { disconnected part }
$$

where $\alpha, \beta$ are SU(3) labels and $q$ is the meson four-momentum. 
single equation to evaluate $\pi \mathrm{N}, \mathrm{KN}, \overline{\mathrm{KN}}$ and $\pi \Sigma$ scattering lengths, we used the sum rules developed by ref. [3] who found that the sum rules for each of these cases differed from the other due to crossing symmetry. The other difference is a different use of experimental data as will be seen below.

To evaluate the dispersion integrals we make appropriate approximations to $\operatorname{Im} T$. For $\left.\pi \mathrm{N})_{I=\frac{1}{2}, \frac{3}{2}}, \mathrm{KN}\right)_{I=0,1}$ and $\left.\pi \Sigma\right)_{I=2}$ we make the elastic unitarity approximation

$$
\operatorname{Im} T=\left(M^{2} x^{2}-m^{2}\right)^{\frac{1}{2}} a^{2} .
$$

Here $M, m$ are the baryon and meson masses respectively, and $x$ is the integration variable defined by

$$
q_{\mu}=x p_{\mu}, \quad \quad p=q=0
$$

where $p_{\mu}$ and $q_{\mu}$ are the baryon and meson four-momenta. With the approximation eq. (1), the dispersion integrals for $\left.\pi N)_{\frac{1}{2}}, \frac{3}{2}, K N\right)_{0,1}$ and $\left.\pi \Sigma\right)_{2}$ were evaluated in close form from threshold to infinity. For $\left.K N)_{0,1}, \pi \Sigma\right)_{0,1}$ and $\pi \Lambda$, where inelastic channels open below threshold couple to the elastic channel, we use the $K$-matrix formalism [6] as an experimental input to evaluate $\operatorname{Im} T$. For the $\overline{\mathrm{K}} \mathrm{N}$ processes we use the $K$-matrix elements given by Martin and Ross [7]. Their parametrization involved a unique nine-parameter, zero-range, s-wave fit to experimental data for $\mathrm{K}^{-} \mathrm{p}$ and $\mathrm{K}_{2}^{0} \mathrm{p}$ reactions below $280 \mathrm{MeV}$. Their results are in close agreement with the parametrization of Martin and Sakitt [8], who used $\mathrm{K}^{-} \mathrm{p}$ reaction data only. Due to the large uncertainties in the values of the $K$-matrix obtained in ref. [7] for $\pi \Sigma$ and $\pi \Lambda$ elastic scattering we used the values given in ref. [8]. In each of these cases we evaluate the dispersion integrals numerically $*$ from the lowest inelastic threshold (which corresponds to $x=\left(M_{\mathrm{i}}+m-M\right) / M$ where $M_{\mathrm{i}}$ is the total rest mass of the particles contributing to the inelastic channel) to $x=100$.

To determine $\mu_{0}$ we used the symmetric amplitudes

$$
\begin{aligned}
& T^{(+)}(\pi \mathrm{N}), \\
& {\left[T\left(\mathrm{~K}^{+} \mathrm{p}\right)+T\left(\mathrm{~K}^{-} \mathrm{p}\right)\right],} \\
& {\left[T\left(\mathrm{~K}^{0} \mathrm{p}\right)+T\left(\overline{\mathrm{K}}^{0} \mathrm{p}\right)\right],}
\end{aligned}
$$

In each case we use experimental data for the real part of the scattering length, thus obtaining three different determinations of $\mu_{0}$. For $\left.\pi N\right)_{\frac{1}{2}}, \frac{3}{2}$ we used the average of the experimental determinations $* *$ of refs. $[9,10]$. For $\mathrm{KN})_{0}$ we used the average

* The numerical integrations were performed at the University of Michigan, Physics Department AEC-HEP computer. The integration programme was furnished by Mr. R. Carroll.

** These are listed in table 1 . 
Table 1

\begin{tabular}{lcrrrr}
\hline & $\begin{array}{l}\text { Soft } \\
\text { meson }\end{array}$ & \multicolumn{1}{l}{$\begin{array}{l}\text { v. Hippel and } \\
\text { Kim }\end{array}$} & \multicolumn{1}{l}{$\begin{array}{l}\text { This } \\
\text { work }\end{array}$} & Experiment & Reference \\
\hline$\pi \mathrm{N})_{\frac{1}{2}}$ & 0.15 & $0.14 \pm 0.01$ & $0.16 \pm 0.01$ & $0.18 \pm 0.01$ & {$[9,10]$} \\
$\pi \mathrm{N})_{\frac{3}{2}}$ & -0.07 & $-0.10 \pm 0.01$ & $-0.10 \pm 0.01$ & $-0.10 \pm 0.01$ & {$[9,10]$} \\
$\mathrm{KN})_{0}$ & 0 & $-0.01 \pm 0.01$ & $0.07 \pm 0.05$ & $0.04 \pm 0.04$ & {$[11,12]$} \\
$\mathrm{KN})_{1}$ & -0.25 & $-0.22 \pm 0.01$ & $-0.22 \pm 0.01$ & $-0.20 \pm 0.01$ & {$[13,14]$} \\
$\overrightarrow{\mathrm{KN}})_{0}$ & 0.37 & $-1.72 \pm 0.13$ & $-1.20 \pm 0.1$ & $1.22 \pm 0.03$ & {$[7]$} \\
$\overline{\mathrm{KN}})_{1}$ & 0.12 & $-0.09 \pm 0.03$ & $-0.07 \pm 0.02$ & $-0.05 \pm 0.03$ & {$[7]$} \\
$\pi \Sigma)_{0}$ & 0.33 & $0.60 \pm 0.1$ & $0.33 \pm 0.04$ & $0.27 \pm 0.02$ & {$[8]$} \\
$\pi \Sigma)_{1}$ & 0.17 & $0.13 \pm 0.07$ & $0.16 \pm 0.09$ & $0.20 \pm 0.02$ & {$[8]$} \\
$\pi \Sigma)_{2}$ & -0.17 & & $-0.11 \pm 0.04$ & & \\
$\pi \Lambda$ & 0 & & $0.02 \pm 0.01$ & $0.09 \pm 0.05$ & {$[8]$} \\
\hline
\end{tabular}

Values of the real part of the meson-baryon scattering lengths in units of the pion mass obtained in the soft meson approximation in the work of von Hippel and Kim and in this work. We also exhibit the experimental results and their references.

of the determinations of refs. [11, 12], for $\mathrm{KN})_{1}$ the average of refs. [13, 14] and for $\overline{\mathrm{K}} \mathrm{N})_{0,1}$ we used the data of ref. [7]. Thus from the reactions (2a)-(2c) we obtain

$$
\begin{aligned}
& \mu_{0}=337 \pm 34 \mathrm{MeV}, \\
& \mu_{0}=232 \pm 28 \mathrm{MeV}, \\
& \mu_{0}=291 \pm 35 \mathrm{MeV},
\end{aligned}
$$

respectively, averaging

$$
\mu_{0}=290 \pm 32 \mathrm{MeV}
$$

Using (4) the pion-nucleon $\sigma$-term is found [2] to be $30 \pm 2 \mathrm{MeV}$. On the other hand. ref. [2] used $\mathrm{KN})_{0,1}$ and $\left.\overline{\mathrm{K} N}\right)_{1}$ elastic scattering reactions only and for the $K$-matrix elements they used Kim's parametrization obtaining $\mu_{0}=215 \mathrm{MeV}$.

Using the value of $\mu_{0}$ obtained in (4) we go back and calculate the real part of the meson-baryon scattering lengths; the results are given in table 1 . If we compare these results with those obtained in ref. [2] we notice that both results show the same qualitative agreement with experiment. However, our value for $\overline{\mathrm{K}} \mathrm{N})_{0}$ scattering 
length is about $50 \%$ smaller in magnitude and shows only a few percent deviation from experiment. Similarly, our $\pi \mathrm{N}$ results indicate a closer agreement with experi ment than the values obtained in ref. [2].

I am grateful to Professor Y. Tomozawa for numerous suggestions during the process of this work and to Dr. M.M. Bakri for reading the manuscript. It is a pleasure to thank Professors Abdus Salam and P. Budini, the International Atomic Energy Agency and UNESCO for hospitality extended to the author at the International Centre for Theoretical Physics, Trieste, where this work was completed.

\section{REFERENCES}

[1] S. Fubini and G. Furlan, Ann. of Phys. 48 (1968) 322.

[2] F. von Hippel and J.K. Kim, Phys. Rev. D1 (1970) 151.

[3] Shafik J. Hakim, Univ. of Michigan preprint 71-21 (1971).

[4] Y. Tomozawa, Nuovo Cimento 46 (1966) 707.

[5] M. Gell-Mann and M. Lévy, Nuovo Cimento $16(1960) 705$.

[6] R.H. Dalitz, Rev. Mod. Phys. 23 (1961) 471.

[7] A.D. Martin and G. Ross, Nucl. Phys. B16 (1970) 479.

[8] B.R. Martin and M. Sakitt, Phys. Rev. 183 (1969) 1352.

[9] V.K. Samaranayake and W.S. Woolcock, Lund Conf. 1969.

[10] G. Höhler et al., Z. Phys. 229 (1969) 217.

[11] S. Goldhaber et al., Phys. Rev. Letters 9 (1962) 135.

[12] V.J. Stenger et al., Phys. Rev. 134B (1964) 1111.

[13] A.D. Martin and R. Perrin, Nucl. Phys. B10 (1969) 125.

[14] B.R. Martin et al., Lund. Conf., 1969.

[15] J.K. Kim, Phys. Letters 19 (1967) 1074. 